\title{
A Escala de Respostas Socialmente Desejáveis-5: Validação numa Amostra Escolar de Jovens Portugueses
}

\author{
The Socially Desirable Response Set-5: Validation among a School Sample of \\ Portuguese Youths
}

\author{
Pedro Pechorro $^{1}$, Cristina Nunes ${ }^{2}$, Rui Abrunhosa Gonçalves ${ }^{3}$, Saul Neves Jesus ${ }^{4}$ \\ e Mário R. Simões ${ }^{5}$
}

\begin{abstract}
Resumo
O objetivo do presente estudo consistiu na validação da Escala de Respostas Socialmente Desejáveis - 5 (SDRS-5) numa amostra escolar $(N=543)$ de jovens portugueses de ambos os sexos. A SDRS-5 apresenta resultados adequados do ponto de vista psicométrico, nomeadamente em termos da sua estrutura fatorial unidimensional, consistência interna, validade convergente, validade discriminante, validade critério e validade de grupos-conhecidos. Foi evidenciada invariância de medida entre rapazes e raparigas. As propriedades psicométricas identificadas justificam a utilização da SDRS-5 com jovens portugueses em contexto escolar.
\end{abstract}

Palavras-chave: avaliação, desejabilidade social, adolescencia, validação

\begin{abstract}
The main aim of the present study was to validate the Socially Desirable Response Set -5 (SDRS-5) among a school sample $(N=543)$ of male and female Portuguese youths. The SDRS-5 demonstrated good results, namely in terms of its one-factor structure, internal consistency, convergent validity, discriminant validity, criterion-related validity and known-groups validity. Cross-gender measurement invariance was demonstrated. The psychometric properties that were found mostly justify the use of the SDRS-5 among Portuguese adolescents from a school context.
\end{abstract}

Keywords: assessment, social desirability, adolescence, validation

A presente investigação foi financiada pela FCT (Bolsa SFRH/BPD/86666/2012).

\footnotetext{
${ }^{1}$ Escola de Psicologia, Universidade do Minho. Campus de Gualtar, 4710-057 Braga, Portugal. Tel.: 253604267. E-mail: ppechorro@gmail.com

${ }^{2}$ Centro de Investigação em Psicologia (CIP/UAL) e Universidade do Algarve. Campus de Gambelas, Edifício 1, 8005-139 Faro,

Portugal. E-mail: csnunes@ualg.pt

${ }^{3}$ Escola de Psicologia, Universidade do Minho. Campus de Gualtar, 4710-057 Braga, Portugal. Tel.: 253604267. E-mail: rabrunhosa@psi.uminho.pt

${ }^{4}$ Centro de Investigação sobre o Espaço e as Organizações, Universidade do Algarve. Campus de Gambelas, Edifício 9, 8005-139 Faro, Portugal. Tel.: 289244406. E-mail: snjesus@ualg.pt

${ }^{5}$ PsyAssessmentLab, Faculdade de Psicologia e de Ciências da Educação, Universidade de Coimbra. Rua do Colégio Novo, 3000 115 Coimbra, Portugal. Tel.: 239851450. E-mail: simoesmr@fpce.uc.pt 


\section{Introdução}

A desejabilidade social pode ser definida como a tendência de alguns sujeitos em responder a uma questão comunicando uma resposta que consideram mais aceitável socialmente que a resposta verdadeira de forma a projetarem uma imagem mais favorável deles próprios, evitando dessa forma avaliações negativas por parte dos outros (Lavrakas, 2008). A desejabilidade social é considerada uma das fontes de erro (enviesamento) relacionadas com os participantes em investigações científicas dado que consiste em sobre relatar comportamentos ou atitudes socialmente desejáveis e na consequente minimização de comportamentos ou atitudes socialmente indesejáveis. Tal enviesamento é particularmente preocupante quando se trata de respostas a questionários de avaliação psicológica porque dificulta significativamente a medição dos traços de personalidade que os instrumentos psicométricos pretendem avaliar e distorce a relação entre as variáveis em estudo (Bäckström \& Björklund, 2013; Nederhof, 1985).

Para avaliar a tendência dos participantes em dar respostas socialmente desejáveis foram expressamente desenvolvidos ao longo dos anos diversos instrumentos psicométricos com o objetivo de detetar e controlar tais respostas. Uma questão importante de investigação relaciona-se com o facto de diferentes medidas de desejabilidade social apresentarem somente correlações de baixas a moderadas umas com as outras (Holtgraves, 2004). Tal aponta para a possibilidade de terem subjacentes conceptualizações diferentes do constructo da desejabilidade social. Outra questão importante tem a ver com diferenças individuais na suscetibilidade em dar respostas socialmente desejáveis. Alguns autores (e.g., Crutzen \& Goritz, 2010; Zerbe \& Paulhus, 1987) consideram que se trata principalmente de uma caraterística de personalidade dos participantes, não uma caraterística dos itens, que pode funcionar como um processo intencional de gestão de impressões (impression management) ou um processo inconsciente de auto-engano (self-deception).

A principal referência de escalas que medem desejabilidade social é a Marlowe-Crowne Social Desirability Scale (MCSDS, Crowne, \& Marlowe,
1960). Esta medida clássica constituída por 33 itens dicotómicos (Verdadeiro/Falso) relativamente subtis em termos de respostas socialmente desejáveis que foram concebidos para serem relativamente livres de traços psicopatológicos ao mesmo tempo que ao nível das normas sociais refletiam comportamentos desejáveis com baixa probabilidade de ocorrência e comportamentos indesejáveis com uma alta probabilidade de ocorrência (Johnston, Wright, \& Weinman, 1995). Utilizando uma amostra de estudantes universitários, Crowne e Marlowe (1960) obtiveram um valor de consistência interna por coeficiente Kuder-Richardson-20 (KR-20)= .88 e uma fiabilidade temporal de .89 . $\mathrm{Na}$ sequência do trabalho destes investigadores, outros estudos analisaram as propriedades psicométricas da MCSDS levando ao desenvolvimento de versões breves aperfeiçoadas através de análise fatorial.

Algumas versões breves da MCSDS concebidas ao longo dos anos destacam-se pelo seu contributo para a literatura da especialidade. Reynolds (1982), recorrendo a uma amostra de estudantes universitários $(N=608)$, efetuou análise de componentes principais e correlações itemtotal da MCSDS, tendo identificado três fatores que designou por A (11 itens), B (12 itens) e C (13 itens) com adequada consistência interna (entre .74 e .76). Ballard (1992), utilizando uma amostra de estudantes universitários $(N=399)$, recorreu a análise de componentes principais para criar diversas versões curtas da MCSDS. A sua versão curta que se tornou mais popular ficou conhecida como MCSDS-SF, sendo constituída por uma estrutura unidimensional de 13 itens e adequada consistência interna (.70). De salientar que na maioria dos estudos recentes com a MCSDS e suas versões breves não se encontram diferenças estatisticamente significativas entre sexos, grupos etários e educacionais (e.g., Andrews \& Meyer, 2003; Barger, 2002; Johnson, Fendrich, \& Mackesy-Amiti, 2012).

Hays, Hayashi e Stewart (1989) estabeleceram o objetivo de construir uma medida de desejabilidade social excecionalmente curta que demorasse menos de um minuto a preencher de forma a poder ser utilizada rotineiramente em avaliações sem significativo consumo de tempo. Para conseguir o seu objetivo recorreram a duas 
amostras ( $N=614$ e $N=3053)$ de pacientes externos e propuseram-se reduziram o número de itens da forma A da MCSDS (Reynolds, 1982), alterando o formato dos itens de dicotómico para ordinal de cinco pontos de forma a melhorar a sensibilidade da medida. Os 5 itens com as maiores correlações item-total foram incluídos na nova medida que foi designada por Escala de Respostas Socialmente Desejáveis - 5 (SDRS-5). Em termos de propriedades psicométricas os alfas de Cronbach tiveram uma amplitude de .66 a .68, a fiabilidade teste-reteste a um mês foi de .75 e as correlações item-total tiveram uma amplitude de .35 a .49 . Segundo Hays et al. (1989), outra vantagem subjacente à utilização da SRDS-5 baseia-se no facto dos itens poderem ser combinados aleatoriamente com os itens de outras escalas de forma a maximizar a validade da escala mesmo que tenham formatos de resposta diferentes (e.g., itens ordinais de 4 pontos), e ser posteriormente dicotomizados (Totalmente verdadeiro $=1$, restantes opções de resposta $=0$ ).

Pechorro, Ayala-Nunes, Oliveira, Nunes e Gonçalves (2016) examinaram as propriedades psicométricas da SDRS-5 numa amostra forense $(N=324)$ de rapazes e raparigas detidos nos oito Centros Educativos do Ministério da Justiça existentes em Portugal. Estes autores propuseramse examinar a estrutura fatorial da SDRS-5, algo que ainda não havia sido feito. Os resultados revelaram uma estrutura unifatorial com adequada consistência interna em rapazes e raparigas. Todavia, não foi examinada a invariância de medida, o que potencialmente enfraquece as interpretações das comparações efetuadas entre os sexos. A validade convergente com a MCSDS-SF e a validade discriminante com medidas de empatia e de traços psicopáticos também apresentaram resultados adequados.

O principal objetivo desta presente investigação foi a validação da versão portuguesa da SDRS-5 numa amostra escolar de adolescentes portugueses de ambos os sexos. Tendo em mente a revisão de literatura efetuada acima, colocámos as seguintes hipóteses de investigação relativamente a este instrumento de avaliação. Assim a SDRS-5 apresenta: (1) estrutura unidimensional que indica invariância de medida entre os sexos, (2) adequada consistência interna examinada através de alfa de Cronbach e do coeficiente ómega, (3) adequada validade convergente (e.g., com base na relação com outra medida de avaliação da desejabilidade social) e validade discriminante (e.g., relação com medidas de agressividade e impulsividade), (4) adequada validade de critério (e.g., com consumo de álcool, de drogas), e (5) adequada validade relativa a grupos-conhecidos.

\section{Método}

\section{Participantes}

A amostra de 543 participantes $(M=15.65$ anos; $D P=1.84$ anos; amplitude $=12-20$ anos, subdividida em rapazes $(n=269 ; M=15.80$ anos; $D P=1.81$ anos; amplitude $=12-20$ anos) e raparigas $(n=274 ; \quad M=15.50 \quad$ anos; $\quad D P=1.86 \quad$ anos; amplitude $=12-20$ anos), foi recolhida em estabelecimentos públicos de ensino da grande Lisboa, Algarve e de Coimbra situados em zonas urbanas. Cerca de $85 \%$ dos participantes tinham nacionalidade portuguesa, $6,3 \%$ eram nacionais de outros países da Europa, 3.1\% eram nacionais do Brasil, $1.5 \%$ eram nacionais de países africanos e $4.1 \%$ tinham outras nacionalidades. Cerca de $89.5 \%$ dos participantes classificaram-se como europeus brancos e $10.5 \%$ classificaram-se como pertencendo a minorias étnicas. Os participantes concluíram em média 9 anos de escolaridade $(D P=1.47 ; \quad$ amplitude=4-12). Não foram encontradas diferenças estatisticamente significativas entre rapazes e raparigas relativamente a idade $(F=3.52 ; p=.06)$, anos de escolaridade completados $(F=5.53 ; p=.12)$ e etnia $\left(\chi^{2}=6.69, p=.12\right)$.

\section{Medidas}

A SDRS-5 (Socially Desirable Response Set5; Hays et al., 1989) é uma medida autorresposta em formato breve constituída por 5 itens que avalia a desejabilidade social. Cada item é cotado numa escala de 5 pontos, de Totalmente verdadeiro a Totalmente falso, após se ter efetuado a reversão dos itens 2,3 e 4 . Pontuações mais altas indicam níveis de desejabilidade social mais altos. Na presente investigação foi utilizada a versão portuguesa da SDRS-5 (Pechorro, AyalaNunes, Oliveira et al., 2016). Na secção Resultados é apresentada a consistência interna 
por alfa de Cronbach e por coeficiente ómega obtidos na presente investigação.

A MCSDS-SF (Marlowe-Crowne Social Desirability Scale-Short Form; Ballard, 1992) foi derivada a partir da escala clássica de MarloweCrowne (Crowne \& Marlowe, 1960). É provavelmente a mais conhecida de todas as versões breves existentes. A MCSDS-SF é cotada somando os itens dicotómicos (Falso/Verdadeiro) que a compõem após se ter efetuado a reversão dos itens apropriados. Pontuações mais elevadas indicam níveis mais altos de respostas socialmente desejáveis. Na presente investigação foi utilizada a versão portuguesa da MCSDS-SF (Pechorro, Vieira, Poiares, \& Maroco, 2012). O coeficiente KR-20 na presente investigação foi de .67 .

O Questionário de Agressividade ReativaProativa (Reactive-Proactive Aggression Questionnaire - RPQ; Raine et al., 2006) é uma medida de autorresposta constituída por 23 itens que distingue entre agressividade reativa $\mathrm{e}$ proativa. Cada item é cotado em escala ordinal de 3 pontos, de Nunca a Frequentemente. O RPQ pode ser utilizado com adolescentes e jovens adultos. Para além da pontuação total podem-se obter também as pontuações nas duas dimensões que constituem o RPQ. Pontuações mais elevadas indicam maior presença das caraterísticas em questão. Na presente investigação foi utilizada a versão portuguesa do RPQ (Pechorro, AyalaNunes, Kahn, \& Nunes, 2018). O coeficiente alfa na presente investigação foi de .86 .

O Dispositivo de Despiste de Processo Antissocial (Antisocial Process Screening DeviceAPSD; Frick \& Hare, 2001) versão de autorresposta (APSD-SR) foi concebido para medir traços psicopáticos em adolescentes. É composto por 20 itens numa escala ordinal de três pontos, de Falso a Totalmente verdade. O APSDSR tem uma estrutura tridimensional composta pelas dimensões Traços Calosos, Impulsividade e Narcisismo. Pode também calcular-se uma pontuação total. Pontuações mais elevadas indicam maior presença de traços psicopáticos. $\mathrm{Na}$ presente investigação foi utilizada a versão portuguesa do APSD-SR (Pechorro, Hidalgo, Nunes, \& Jiménez, 2016). O coeficiente alfa na presente investigação foi de .77 .

O Inventário de Traços Psicopáticos em Jovens (Youth Psychopathic Traits Inventory short version-YPI-S; van Baardewijk et al., 2010) é um instrumento breve concebido para medir traços psicopáticos em adolescentes. Os 18 itens que o constituem são cotados numa escala ordinal de 4 pontos, de Não se aplica a Aplica-se muito bem. O YPI-S tem uma estrutura tridimensional semelhante à do YPI original composta pelas dimensões comportamental, afetiva e interpessoal. Pontuações mais elevadas indicam maior presença dos traços em questão. Na presente investigação foi utilizada a versão portuguesa do YPI-S (Pechorro, Ribeiro da Silva, Andershed, Rijo, \& Gonçalves, 2016). O coeficiente alfa na presente investigação foi de .92 .

O Inventário de Traços Calosos/Insensibilidade Emocional (Inventory of Callous-Unemotional Traits-ICU; Essau, Sasagawa, \& Frick, 2006) é uma medida de autorresposta composta por 24 itens desenhada para medir traços calosos/insensibilidade emocional (frieza emocional/ausência de emocionalidade) em jovens. Os itens são cotados numa escala ordinal de 4 pontos, de Discordo totalmente a Concordo totalmente. O ICU tem uma estrutura tridimensional composta pelas dimensões Calosidade/Frieza Emocional, Insensibilidade Emocional e Indiferença Emocional. Pode também ser calculada a pontuação total. Pontuações mais elevadas indicam maior presença dos traços em questão. $\mathrm{Na}$ presente investigação foi utilizada a versão portuguesa do ICU (Pechorro, Ray, Barroso, Maroco, \& Gonçalves, 2016). O coeficiente alfa na presente investigação foi de .88 .

A Escala de Impulsividade de Barratt - versão 11 (Barratt Impulsiveness Scale version 11 - BIS11; Patton et al., 1995) é uma medida de autorresposta composta por 30 itens projetada para medir impulsividade. Os itens são cotados numa escala ordinal de 4 pontos, de Raramente/Nunca a Quase sempre/Sempre. A BIS-11 tem seis subescalas que correspondem a seis fatores de primeira ordem, nomeadamente: Atenção (5 itens), Instabilidade cognitiva (3 itens), Motora (7 itens), Perseverança (4 itens), Autocontrolo (6 itens) e Complexidade cognitiva (5 itens) que deverão convergir em três fatores de segunda ordem, nomeadamente: Impulsividade atencional, Impulsividade motora e Impulsividade de não-planeamento. Pontuações mais elevadas 
indicam maior presença de impulsividade. $\mathrm{Na}$ presente investigação utilizou-se a versão reduzida da BIS-11 composta pelos 18 itens constituintes dos fatores de primeira ordem Atenção, Motora e Autocontrolo (Pechorro, Oliveira, Gonçalves, \& Jesus, 2018). O coeficiente alfa na presente investigação foi de .87 .

O Inventário de Personalidade Narcísica-13 (Narcissistic Personality Inventory-13 - NPI-13; Gentile et al., 2013) é uma medida curta de narcisismo. Os 13 itens do NPI-13 mantêm o formato dicotómico do instrumento original. A estrutura fatorial do NPI-13 possui três dimensões, nomeadamente: Liderança/Autoridade (L/A), Grandiosidade/Exibicionismo (G/E) e Empossamento/Exploratividade (E/E). Pode também calcular-se a pontuação total. Pontuações mais elevadas indicam níveis de narcisismo mais elevados. Na presente investigação foi utilizada a versão portuguesa do NPI-13 (Pechorro, Gentile, Ray, Nunes, \& Gonçalves, 2016; Pechorro, Nunes, Gonçalves, Simões, \& Oliveira, 2019). O coeficiente alfa na presente investigação foi de .83 .

A Escala de Ansiedade Social para Adolescentes (Social Anxiety Scale for Adolescents - SAS-A; La Greca \& Lopez, 1998) é uma medida de autorresposta, constituída por 22 itens (dos quais 4 são itens neutros não cotados), que avalia as experiências de ansiedade social dos adolescentes no contexto das relações com os seus pares. Os itens são avaliados segundo uma escala ordinal de 5 pontos, que vai de De forma nenhuma a Todas as vezes. Para além da pontuação total pode-se obter também as pontuações nas três dimensões que constituem a SAS-A, nomeadamente: FNE, SAD-Novo e SAD-Geral. Pontuações mais elevadas indicam níveis de ansiedade social mais elevados. Na presente investigação foi utilizada a versão portuguesa da SAS-A (Pechorro, Ayala-Nunes, Nunes, Maroco, \& Gonçalves, 2016). O coeficiente alfa na presente investigação foi de .92 .

A Escala de Empatia Básica (Basic Empathy Scale - BES; Jolliffe \& Farrington, 2006) é uma medida de autorresposta constituída por 20 itens, desenhada para medir duas dimensões da empatia em jovens: empatia afetiva e empatia cognitiva. Cada item é cotado em escala ordinal de 5 pontos, de Discordo totalmente a Concordo totalmente. Para além da pontuação total pode-se obter também as pontuações nas duas dimensões que constituem a BES. Pontuações mais elevadas indicam níveis de empatia mais elevados. $\mathrm{Na}$ presente investigação foi utilizada a versão portuguesa da BES (Pechorro, Ray, Salas-Wright, Maroco, \& Gonçalves, 2015). O coeficiente alfa na presente investigação foi de .91 .

Os 15 critérios para o diagnóstico de Perturbação do Comportamento de acordo com os critérios oficiais do DSM-5 (American Psychiatric Association, 2013) foram utilizados para criar uma escala de autorresposta (ver e.g., Skilling, Quinsey, \& Craig, 2001). Os 15 itens (codificados $0=$ Não, $1=$ Sim) foram somados para obter uma pontuação total, de forma que pontuações mais altas indicam maior presença de sintomas de perturbação de comportamento. O coeficiente KR20 na presente investigação foi de .81 .

Foi construído um questionário sociodemográfico para descrever as caraterísticas dos participantes, que incluiu variáveis como idade, sexo, escolaridade e etnia. Este questionário incluiu também itens ordinais tipo Likert de 5 pontos sobre consumo de álcool, drogas leves (cannabis), drogas duras (ecstasy, cocaína, heroína) e sexo desprotegido (i.e., sem preservativo) durante os últimos 12 meses.

\section{Procedimentos}

Foi previamente solicitada autorização para utilizar o instrumento em Portugal. Durante o processo de tradução e adaptação da SDRS-5 foram seguidas as recomendações estabelecidas internacionalmente (Hambleton, Merenda, \& Spielberger, 2005). O primeiro autor deste artigo e um colega efetuaram a tradução da escala. De seguida um tradutor bilingue fez a respetiva retroversão para inglês, que foi então comparada com o instrumento original. Posteriormente realizou-se um estudo pré-teste para analisar a qualidade da tradução, detetar problemas eventuais problemas e aperfeiçoar a linguagem de forma a torná-la mais facilmente entendível, chegando-se assim à versão final (ver Pechorro, Ayala-Nunes, Oliveira et al., 2016, para uma descrição pormenorizada).

A recolha dos questionários decorreu em escolas básicas/secundárias da região da grande 
Lisboa, Algarve e de Coimbra após se ter obtido autorização por parte da Direção-Geral de Educação (DGE). Foi entregue um termo de consentimento assinado pelo encarregado de educação de cada aluno autorizando a participação na investigação. A aplicação decorreu em contexto de grupo. A taxa de participação foi de aproximadamente $85 \%$. Foram excluídos os participantes que estavam fora do intervalo etário estabelecido, que entregaram questionários não utilizáveis (e.g., não preenchidos, incompletos, ilegíveis), com perturbações mentais diagnosticadas, a tomar medicação psiquiátrica, com perturbações da aprendizagem ou inseridos em medidas de educação especial.

Os dados foram inseridos e analisados utilizando o software SPSS v25 (IBM SPSS, 2017) e o software EQS 6.3 (Bentler \& Wu, 2015). A análise da estrutura fatorial da SDRS-5 foi efetuada no software EQS com estimação Maximum Likelihood (ML). Os índices de ajustamento calculados incluíram: Qui-quadrado de Satorra-Bentler/graus de liberdade, CFI (Comparative Fit Index), IFI (Incremental Fit Index), RMSEA (Root Mean Square Error of Approximation). Valores CFI $\geq .90$ e RMSEA $<.10$ indicam ajustamento adequado; valores de CFI $\geq .95$ e RMSEA $\leq .06$ indicam um ajustamento bom (Byrne, 2006). Um valor de IFI $\geq .90$ é considerado aceitável. A AFC foi efetuada diretamente nos itens utilizando valores de cargas fatoriais $\geq .45$. Nos modelos testados o Coeficiente de Mardia situou-se acima de 5, indicando uma distribuição não normal, pelo que se optou pela utilização de matriz de correlações policóricas com métodos de estimação robustos nos itens ordinais dado que proporcionam melhores resultados (Byrne, 2006; Maroco, 2014; Satorra \& Bentler, 1988). A invariância de medida foi testada com as amostras masculina e feminina. $\mathrm{O}$ teste de diferença de $\mathrm{SB} \chi^{2}$ foi utilizado determinar o ajustamento do modelo, além do $\triangle$ CFI e $\triangle$ RMSEA (Byrne \& van de Vijver, 2010; Chen, 2007; Cheung \& Rensvold, 2002. O ficheiro Excel (http://www.econ.upf.edu/ satorra/) fornecido por Bryant e Satorra (2012) foi utilizado para proceder aos testes de diferença de quiquadrado de Satorra-Bentler.
ANOVA foi utilizada para comparar os grupos masculino e feminino. Correlações Pearson foram utilizadas para analisar as associações entre variáveis escalares e correlações Spearman para analisar a relação entre variáveis ordinais e variáveis escalares (Leech, Barrett, \& Morgan, 2015). Consideraram-se correlações fracas as correlações entre 0 e .20 , correlações moderadas entre .20 e .50 , e correlações fortes acima de .50 (Ferguson, 2009). A consistência interna por alfa de Cronbach e por ómega foi considerada adequada se acima de .70 e as correlações item-total corrigidas foram consideradas adequadas se acima de .30 (Dunn, Baguley, \& Brunsden, 2014; Nunnally, \& Bernstein, 1994; Urbina, 2014).

\section{Resultados}

O primeiro passo do tratamento de dados consistiu na confirmação da estrutura fatorial unidimensional. Os seguintes índices de ajustamento foram obtidos para a amostra total $\mathrm{SB} \chi^{2} / \mathrm{gl}=6.01, \quad p \leq .01, \quad \mathrm{IFI}=.97, \quad \mathrm{CFI}=.97$, RMSEA $=.07$ [.04-.10], amostra masculina $\mathrm{SB} \chi^{2} / \mathrm{gl}=4.08, \quad p \leq .01, \quad \mathrm{IFI}=.97, \quad \mathrm{CFI}=.97$, RMSEA $=.08$ [.05-.11] e amostra feminina $\mathrm{SB} \chi^{2} / \mathrm{gl}=1.32, \quad p=.25, \quad \mathrm{IFI}=.99, \quad \mathrm{CFI}=.99$, RMSEA $=.03$ [.00-.07]. Tais valores indicam que se obtiveram ajustamentos de adequados a bons. No Quadro 1 podem-se observar as saturações fatoriais dos itens e as correlações item-total corrigidas para a amostra total, masculina e feminina.

Seguidamente testamos a invariância de medida. O modelo base foi testado em termos de invariância métrica (fraca) e invariância escalar (forte), apresentando-se os resultados no Quadro 2. Os valores de $\triangle \mathrm{SB} \chi 2(\mathrm{df})$ foram sempre não significativos, e se considerarmos também os critérios de Chen (2007) e de Cheung e Rensvold (2002), nomeadamente, $\triangle$ CFI abaixo de .01, $\triangle$ RMSEA abaixo de .015 , existe suporte para invariância entre rapazes e raparigas.

De seguida calcularam-se os valores de consistência interna, tendo-se obtido na amostra masculina um alfa de Cronbach de .72 e na amostra feminina um alfa de Cronbach de .71. Calculou-se também o coeficiente ómega, tendo a 
Quadro 1. Saturações fatoriais e correlações item-total para o modelo unidimensional da SDRS-5

\begin{tabular}{lcc}
\hline Itens & $\begin{array}{c}\text { Saturações } \\
\text { T/M/F }\end{array}$ & $\begin{array}{c}\text { CITC } \\
\text { T/M/F }\end{array}$ \\
\hline 1. Sou sempre simpático, mesmo com pessoas que são mal-educadas. & $.58 / .54 / .48$ & $.42 / .31 / .27$ \\
2. Já me aproveitei de outras pessoas para meu ganho pessoal. (R) & $.72 / .64 / .68$ & $.52 / .44 / .42$ \\
3. Por vezes tento vingar-me em vez de perdoar e esquecer. (R) & $.68 / .67 / .56$ & $.49 / .52 / .48$ \\
4. Por vezes fico chateado quando não consigo o que quero. (R) & $.63 / .59 / .68$ & $.51 / .50 / .52$ \\
5. Ouço sempre com muita atenção todas as pessoas com quem falo. & $.64 / .57 / .69$ & $.50 / .47 / .54$ \\
\hline Nota. SDRS-5=Escala de Respostas Socialmente Desejáveis & - & $5, \quad$ T/M/F=Total/Masculino/Feminino, \\
CITC=Correlações item-total corrigidas & &
\end{tabular}

Quadro 2. Invariância de medida da SDRS-5

\begin{tabular}{lcccc}
\hline Modelo & $\mathrm{SB} \chi^{2}(\mathrm{df})$ & $\Delta \mathrm{SB} \chi^{2}(\mathrm{df})$ & $\mathrm{CFI}$ & $\begin{array}{c}\text { RMSEA (90\% } \\
\text { C.I.) }\end{array}$ \\
\hline Modelo base (configural) & $28.19(10)$ & -- & .98 & $.07(.04-.10)$ \\
Invariância métrica (fraca) & $37.25(14)$ & $8.98(4)^{n s}$ & .98 & $.07(.04-.09)$ \\
Invariância escalar (forte) & $37.50(15)$ & $9.29(5)^{n s}$ & .98 & $.06(.04-.09)$ \\
\hline
\end{tabular}

Nota. $\mathrm{SB} \chi^{2}(\mathrm{df})=$ Satorra-Bentler chi-square (degrees of freedom), CFI=Comparative Fit Index, RMSEA=Root Mean Square Error of Approximation, C.I.=confidence interval $n s=$ não-significativo

Quadro 3. Validade convergente, discriminante e de critério da SDRS-5

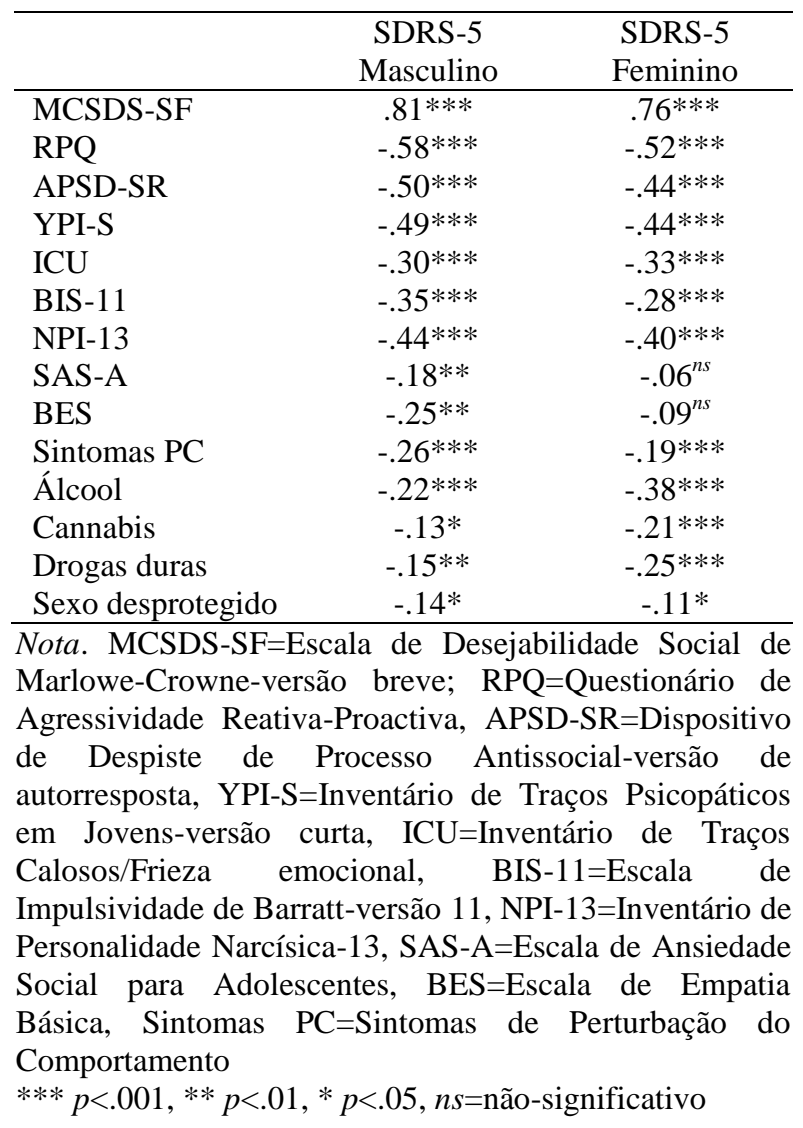

amostra masculina valor de .77 e na amostra feminina um valor de .75 .

A validade convergente, a validade discriminante e a validade de critério são apresentadas no Quadro 3. A validade convergente foi efetuada com a MCSDS-SF. A validade discriminante foi efetuada com o RPQ, o APSD-SR, o YPI-S, o ICU, a BIS-11, o NPI-13, a
SAS-A e a BES. A validade de critério (ou concorrente) foi efetuada com os sintomas de Perturbação de Comportamento, o consumo de álcool, de cannabis, de drogas duras e os comportamentos sexuais desprotegidos (i.e., sexo sem preservativo).

Finalmente, em termos da validade de gruposconhecidos procedemos à comparação entre rapazes e raparigas, não tendo sido encontradas diferenças significativas $(F=3.055, p=.08$, eta parcial $^{2}=.01$, potência $=.42, M_{\text {rapazes }}=12.05, D P$ rapazes $=3.56, M_{\text {raparigas }}=12.49, D P_{\text {raparigas }}=3.60$ ).

\section{Discussão}

O objetivo do presente estudo consistiu na validação da SDRS-5 numa amostra escolar de adolescentes portugueses. Através de análise fatorial confirmatória foi possível comprovar a existência da estrutura unidimensional latente esperada em rapazes e raparigas (West, Taylor, \& $\mathrm{Wu}, 2012)$. As saturações dos itens estiveram sempre acima de .45 e as correlações item-total corrigidas estiveram sempre acima de .30 de acordo com critérios recomendados (Nunnally, \& Bernstein, 1994). Seguidamente foi demonstrada invariância de medida métrica (fraca) e escalar (forte) entre rapazes e raparigas, o que permite a realização de comparações entre estes dois grupos (Chen, 2007). Tal é especialmente importante dado que tanto quanto é do nosso conhecimento é a primeira vez que se demonstrou a invariância da 
SDRS-5. Avaliar a invariância de medida é um aspeto crucial para o desenvolvimento e uso de instrumentos psicométricos, sendo que atualmente é pré-requisito para qualquer estudo que tenha por objetivo avaliar diferenças entre grupos (Byrne \& van de Vijver, 2010).

A consistência interna (fiabilidade) por alfa de Cronbach e por coeficiente ómega revelou valores de adequados para rapazes e raparigas, sempre acima do valor recomendado de .70 (Dunn et al., 2014; Nunnally \& Bernstein, 1994), que são inclusivamente mais elevados que os valores reportados em estudos prévios (Hays et al., 1989; Pechorro, Ayala-Nunes, Oliveira et al., 2016).

A validade convergente com a MCSDS-SF revelou as correlações positivas altas e estatisticamente significativas. Tal já era esperado dado que ambas as medidas partilham o mesmo pool de itens da MCSDS. A validade discriminante com o RPQ, APSD-SR, YPI-S, ICU, BIS-11, NPI-13, SAS-A e BES revelou de uma forma geral correlações negativas ou nãosignificativas conforme o esperado, em linha com investigações prévias (Kämpfe, Penzhorn, Schikora, Dünzl, \& Schneidenbach, 2009; Pechorro, Ayala-Nunes, Oliveira et al., 2016; Ray et al., 2013; Verschuere et al., 2014).

A validade de critério com os sintomas de PC, o uso de álcool, de cannabis, de drogas duras e o sexo desprotegido (i.e., sem preservativo) revelou de uma forma geral correlações negativas ou nãosignificativas conforme o esperado, em linha com investigações prévias (Pechorro, Ayala-Nunes, Oliveira et al., 2016). Finalmente, em termos de validade relativa a grupos-conhecidos, não foram encontradas diferenças entre rapazes e raparigas, $o$ que é consistente com estudos prévios (Johnson et al., 2012; Pechorro, Vieira et al., 2012; Pechorro, Ayala-Nunes, Oliveira et al., 2016).

Os resultados obtidos levam-nos a concluir que a SDRS-5 demonstrou propriedades psicométricas adequadas que justificam a sua utilização com o propósito de medir a desejabilidade social em adolescentes portugueses no contexto escolar de avaliação psicológica. Os benefícios da utilização da SRDS-5 são consideráveis a nível investigativo e de prática clínica dado que as versões breves das escalas têm diversas vantagens, entre as quais: demoram menos tempo a responder logo são menos fatigantes e menos aborrecidas para certas populações (e.g., adolescentes), diminuem a probabilidade de haver itens omissos, aumentam a validade facial na perspetiva dos participantes e são mais representativas do constructo em questão por manterem apenas os itens essenciais após eliminação de itens repetidos ou muito semelhantes (Rammstedt \& Beierlein, 2014).

Devemos, todavia, mencionar algumas limitações da nossa investigação. Teria sido aconselhável a utilização de outras medidas validadas de desejabilidade social que não partilhassem os mesmos itens para examinar a validade convergente das pontuações. Estudos futuros devem ser realizados com vista à obtenção de mais dados de natureza psicométrica sobre o instrumento (e.g., fiabilidade teste-reteste, visando averiguar a estabilidade temporal das pontuações). Dever-se-á igualmente proceder à validação cruzada noutras amostras (e.g., amostras clínicas de adolescentes com diagnóstico de problemas de consumo de álcool e/ou drogas, perturbações emocionais como ansiedade e depressão, ofensores com comportamentos agressivos) de forma a ter confiança de que as propriedades psicométricas se mantêm adequadas.

\section{Referências}

American Psychiatric Association. (2013). Diagnostic and statistical manual of mental disorders (5th ed.). Arlington, VA: American Psychiatric Publishing.

Andrews, P., \& Meyers, R. (2003). MarloweCrowne Social Desirability Scale and short form C: Forensic norms. Journal of Clinical Psychology, 59, 483-492. doi:10.1002/jclp.10136

Bäckström, M., \& Björklund, F. (2013). Social desirability in personality inventories: Symptoms, diagnosis and prescribed cure. Scandinavian Journal of Psychology, 54, 152159. doi:10.1111/sjop. 12015

Ballard, R. (1992). Short forms of the MarloweCrowne Social Desirability Scale. Psychological Reports, 71, 1155-1160. doi:10.2466/PR0.71.8.1155-1160

Barger, S. (2002). The Marlowe-Crowne affair: Short forms, psychometric structure and social 
desirability. Journal of Personality Assessment, 79, 286-305. doi:10.1207/S15327752JPA7902_11

Bentler, P., \& Wu, E. (2015). Supplement to EQS 6.3 for Windows user's guide. Temple City, CA: Multivariate Software.

Byrne, B. (2006). Structural equation modeling with EQS: Basic concepts, applications, and programming. Mahwah, NJ: Lawrence Erlbaum Associates.

Byrne, B., \& van de Vijver, F. (2010). Testing for measurement and structural equivalence in large-scale cross-cultural studies: Addressing the issue of nonequivalence. International Journal of Testing, 10, 107-132. doi:10.1080/15305051003637306

Chen, F. F. (2007). Sensitivity of goodness of fit indexes to lack of measurement invariance. Structural Equation Modeling, 14, 464-504. doi:10.1080/10705510701301834

Cheung, G. W., \& Rensvold, R. B. (2002). Evaluating goodness-of-fit indexes for testing measurement invariance. Structural Equation Modelling, 233-255. doi:10.1207/S15328007SEM0902_5

Crowne, D., \& Marlowe, D. (1960). A new scale of social desirability independent of psychopathology. Journal of Consulting Psychology, 24, 349-354. doi:10.1037/h0047358

Crutzen, R., \& Goritz, A. (2010). Social desirability and self-reported health risk behaviors in web-based research: Three longitudinal studies. BMC Public Health, 10, 720. doi:10.1186/1471-2458-10-720

Dunn, T., Baguley, T., \& Brunsden, V. (2014). From alpha to omega: A practical solution to the pervasive problem of internal consistency estimation. British Journal of Psychology, 105, 399-412. doi:10.1111/bjop.12046

Essau, C. A., Sasagawa, S., \& Frick, P. J. (2006). Callous-unemotional traits in a community sample of adolescents. Assessment, 13, 454469. doi:10.1177/1073191106287354

Ferguson, C. (2009). An effect size primer: A guide for clinicians and researchers. Professional Psychology: Research and Practice, 40, 532-538. doi:10.1037/a0015808

Frick, P., \& Hare, R. (2001). The Antisocial Process Screening Device (APSD): Technical manual. Toronto, ON: Multi-Health Systems.
Gentile, B., Miller, J., Hoffman, B., Reidy, D., Zeichner, A., \& Campbell, W. (2013). A Test of two brief measures of grandiose narcissism: The Narcissistic Personality Inventory-13 and the Narcissistic Personality Inventory-16. Psychological Assessment, 25, 1120-1136. doi:10.1037/a0033192

Hambleton, R., Merenda, P., \& Spielberger, C. (2005). Adapting educational and psychological tests for cross-cultural assessment. Mahwah, NJ: Lawrence Erlbaum Associates.

Hays, R., Hayashi, T., \& Stewart, A. (1989). A five-item measure of Socially Desirable Response Set. Educational and Psychological Measurement, 49, 629-636. doi:10.1177/001316448904900315

Holtgraves, T. (2004). Social desirability and selfreports: Testing models of socially desirable responding. Personality and Social Psychology Bulletin, 30, 161-172. doi:10.1177/0146167203259930

IBM SPSS. (2017). IBM SPSS statistics base 25. Chicago, IL: SPSS.

Jolliffe, D., \& Farrington, D. (2006). Development and validation of the Basic Empathy Scale. Journal of Adolescence, 29, 589-611. doi:10.1016/j.adolescence.2005.08.010

Johnston, M., Wright, S., \& Weinman, J. (1995). Measures in health psychology: A user's portfolio. Windsor (UK): NFER-NELSON Publishing Company Ltd.

Johnson, T. P., Fendrich, M., \& Mackesy-Amiti, M. E. (2012). An evaluation of the validity of the Crowne-Marlowe need for approval scale. Quality \& Quantity, 46, 1883-1896. doi:10.1007/s11135-011-9563-5

Kämpfe, N., Penzhorn, J., Schikora, J., Dünzl, J., \& Schneidenbach, J. (2009). Empathy and social desirability: a comparison of delinquent and non-delinquent participants using direct and indirect measures. Psychology, Crime \& Law, $\quad$ 15(1), 1-17. doi:10.1080/10683160802010640

La Greca, A., \& Lopez, N. (1998). Social anxiety among adolescents: Linkages with peer relations and friendships. Journal of Abnormal Child Psychology, 26, 83-94. doi:10.1023/A:1022684520514 
Lavrakas, P. J. (2008). Encyclopedia of survey research methods. Thousand Oaks, CA: SAGE Publications. doi:10.4135/9781412963947

Leech, N., Barrett, K., \& Morgan, G. (2015). IBM SPSS for intermediate statistics. New York, NY:LEA.

Maroco, J. (2014). Análise de Equações Estruturais: Fundamentos teóricos, software \& aplicações. Pero Pinheiro: ReportNumber.

Nederhof, A. (1985). Methods of coping with social desirability bias: A review. European Journal of Social Psychology, 15, 263-280. doi:10.1002/ejsp.2420150303

Nunnally, J. C., \& Bernstein, I. H. (1994). Psychometric theory ( ${ }^{\text {rd }}$ ed.). New York: McGraw-Hill.

Patton, J. H., Stanford, M. S., \& Barratt, E. S. (1995). Factor structure of the Barratt Impulsiveness Scale. Journal of Clinical Psychology, 51, 768-774. doi:10.1002/10974679(199511)51:6<768::AIDJCLP2270510607>3.0.CO;2-1

Pechorro, P., Vieira, R., Poiares, C., \& Maroco, J. (2012). Contributos para a validação duma versão curta da Escala de Desejabilidade Social de Marlowe-Crowne com adolescentes portugueses. Arquivos de Medicina, 26(1), 1117.

Pechorro, P., Gentile, B., Ray, J. V., Nunes, C., \& Gonçalves, R. (2016). Adaptation of the Narcissistic Personality Inventory among a Portuguese sample of incarcerated juvenile offenders. Psychology, Crime \& Law, 22, 495511. doi:10.1080/1068316X.2016.1168421

Pechorro, P., Ayala-Nunes, L., Nunes, C., Maroco, J., \& Gonçalves, R. (2016). The Social Anxiety Scale for Adolescents: Measurement invariance and psychometric properties among a school sample of Portuguese youths. Child Psychiatry and Human Development, 47, 975-984. doi:10.1007/s10578-016-0627-6

Pechorro, P., Hidalgo, V., Nunes, C., \& Jiménez, L. (2016). Confirmatory factor analysis of the Antisocial Process Screening Device: SelfReport among incarcerated male juvenile offenders. International Journal of Offender Therapy and Comparative Criminology, 60, 1856-1872. doi:10.1177/0306624X15588903
Pechorro, P., Ray, J. V., Salas-Wright, C., Maroco, J., \& Gonçalves, R. A. (2015) Adaptation of the Basic Empathy Scale among a Portuguese sample of incarcerated juvenile offenders. Psychology, Crime and Law, 21, 699-714. doi:10.1080/1068316X.2015.1028546

Pechorro, P., Ayala-Nunes, L., Oliveira, J. P., Nunes, C., \& Gonçalves, R. (2016). Psychometric properties of the Socially Desirable Response Set-5 among incarcerated male and female juvenile offenders. International Journal of Law and Psychiatry, 49, 17-21. doi:10.1016/j.ijlp.2016.05.003

Pechorro, P., Ribeiro da Silva, D., Rijo, D., Gonçalves, R. A., \& Andershed, H. (2017). Psychometric properties and measurement invariance of the Youth Psychopathic Traits Inventory - Short among Portuguese youths. Journal of Psychopathology and Behavioral Assessment, $\quad 39, \quad 486-497$. doi:10.1007/s10862-017-9597-7

Pechorro, P., Oliveira, J. P., Gonçalves, R., \& Jesus, S. (2018). Propriedades psicométricas de uma versão reduzida da Escala de Impulsividade de Barratt - 11 numa amostra escolar de adolescentes portugueses. Revista Iberoamericana de Diagnóstico y Evaluación - e Avaliação Psicológica, 47, 157-170. doi:10.21865/RIDEP47.2.11

Pechorro, P., Nunes, C., Gonçalves, R., Simões, M., \& Oliveira, J. P. (2019). Estudo de validação do Inventário de Personalidade Narcísica - 13 em uma amostra escolar de jovens portugueses. Revista Iberoamericana de Diagnóstico y Evaluación - e Avaliação Psicológica.

Pechorro, P., Ayala-Nunes, L., Kahn, R., \& Nunes, C. (2018). The Reactive-Proactive Aggression Questionnaire: Measurement invariance and reliability among a school sample of Portuguese youths. Child Psychiatry \& Human Development. doi:10.1007/s10578-017-0772-6

Raine, A., Dodge, K., Loeber, R., Gatzke-Kopp, L., Lynam, D., Reynolds, C., ... \& Liu, J. (2006). The Reactive-Proactive Aggression Questionnaire: Differential correlates of reactive and proactive aggression in 
adolescent boys. Aggressive Behavior, 32, 159-171. doi:10.1002/ab.20115

Rammstedt, B., \& Beierlein, C. (2014). Can't we make it any shorter? The limits of personality assessment and ways to overcome them. Journal of Individual Differences, 35, 212220. doi:10.1027/1614-0001/a000141

Ray, J., Hall, J., Rivera-Hudson, N., Poythress, N., Lilienfeld, S., \& Morano, M. (2013). The relation between self-reported psychopathic traits and distorted response styles: A metaanalytic review. Personality Disorders: Theory, Treatment and Research, 4(1), 1-14. doi:10.1037/a0026482

Reynolds, W. (1982). Development of reliable and valid short forms of the Marlowe-Crowne Social Desirability Scale. Journal of Clinical Psychology, 38, 119-125. doi:10.1002/10974679(198201)38:1\%3C119::AIDJCLP2270380118\%3E3.0.CO;2-I

Satorra, A., \& Bentler, P. M. (1988). Scaling corrections for chi square statistics in covariance structure analysis. In American Statistical Association (Ed.), Proceedings of the business and economic sections (pp. 308313). Alexandria, VA: American Statistical Association.

Skilling, T., Quinsey, V., \& Craig, W. (2001). Evidence of a taxon underlying serious antisocial behavior in boys. Criminal Justice and Behavior, 28, 450-470. doi:10.1177/009385480102800404

Urbina, S. (2014). Essentials of psychological testing ( $2^{\text {nd }}$ ed.). Hoboken, NJ: John Wiley \& Sons.

Van Baardewijk, Y., Andershed, H., Stegge, H., Nilsson, K., Scholte, E., \& Vermeiren, R. (2010). Development and tests of short versions of the Youth Psychopathic Traits Inventory and the Youth Psychopathic Traits Inventory-Child Version. European Journal of Psychological Assessment, 26, 122-128. doi:10.1027/1015-5759/a000017

Verschuere, B., Uzieblo, K., De Schryver, M., Douma, H., Onraedt, T., \& Crombez, G. (2014). The inverse relation between psychopathy and faking good: Not response bias, but true variance in psychopathic personality. Journal of Forensic Psychiatry \&
Psychology,

25 , 705-713. doi:10.1080/14789949.2014.952767

West, S., Taylor, A., \& Wu, W. (2012). Model fit and model selection in structural equation modeling. In R. Hoyle (Ed.), Handbook of structural equation modeling (pp. 209-231). New York, NY: The Guilford Press.

Zerbe, W., Paulhus, D. (1987). Socially desirable responding in organizational behavior: A reconception. Academy of Management Review, 12, 250-264. 